\title{
Model Reduction Opportunities in Detailed Simulations of Combustion Dynamics
}

\author{
Ramakanth Munipalli ${ }^{1}$, Zhining Liu $^{2}$ \\ HyPerComp Inc., Westlake Village, CA 91361 \\ Xueyu Zhu ${ }^{3}$ \\ Scientific Computing and Imaging Institute, University of Utah \\ Suresh Menon ${ }^{4}$ \\ Department of Aerospace Engineering, Georgia Institute of Technology \\ Jan S. Hesthaven ${ }^{5}$ \\ Division of Applied Mathematics, Brown University
}

\begin{abstract}
Rocket and gas turbine combustion dynamics involves a confluence of diverse physics and interaction across a number of system components. Any comprehensive, self-consistent numerical model is burdened by a very large computational mesh, stiff unsteady processes which limit the permissible time step, and the need to perform tedious, repeated calculations for a broad parametric range. Predictive CFD models rely on very large scale simulations and advanced hardware. Reduced Basis Methods (RBM) have grown in usage during the past decade, as promising new techniques in making large simulations more accessible. These methods create models with far fewer unknown quantities than the original system, by generating "proper" fundamental solutions and their Galerkin projections, while guaranteeing accuracy and computational efficiency. RBMs seek to reproduce full CFD solutions, rather than solutions to a simplified or linearized set of equations. We present here some recent work in this area, focusing on approaches to model large scale combustor systems. The maturation of methods leading to LES-based turbulent combustion modeling is discussed, and model reduction goals and strategies are explored from the perspective of applicability in real life problems in both gas turbine, as well as rocket engines.
\end{abstract}

\section{Introduction}

The study of combustion dynamics is motivated by the need to understand stability and performance of systems such as rocket engines, ramjets, gas turbines and afterburners (Culick ${ }^{9}$ ), as well as boilers, blast furnaces and oil/gas/coal fired heating units. Combustion instabilities arise when the heat release associated with combustion process provides a driving mechanism for the acoustic oscillations of the enclosed chamber (combustor). These instabilities manifest as pressure and temperature oscillations that have a tendency to grow, leading to catastrophic structural failures. Self-consistent CFD methods to study these phenomena are beset by the great demands upon computational resources that face them.

The commonly used methods to analyze combustion dynamics were developed over many decades, an extensive survey of which may be found in the monograph by Culick [9]. These methods involve first expanding the complete system of Navier-Stokes equations using a two-parameter perturbation series. The resulting equations are recast in terms of perturbation pressure, and a nonlinear second order wave equation is derived. A Galerkin approximation is then used to represent the pressure perturbation in terms of a set of position-dependent basis functions. These methods provide valuable insights in the qualitative aspects of combustion instabilities. However, they have limited

\footnotetext{
${ }^{1}$ Sr. Computational Physicist, Senior Member, AIAA.

${ }^{2}$ Member, AIAA

${ }^{3}$ Post-doctoral Researcher

${ }^{4}$ Professor, Director of Computational Combustion Laboratory, Member, AIAA

${ }^{5}$ Adjunct Professor, presently Professor, EPFL-SB-MATHICSE-MCSS, Lausanne, Switzerland
}

1

American Institute of Aeronautics and Astronautics 
predictive value in a practical design of a combustor due to the many physical and geometric simplifications they contain.

In this paper, we present the conceptual development of models of reduced complexity - variously termed "reduced order" or "reduced basis" methods (see ref [20] for a review) which do not simplify the original set of governing equations. The primary goal of these methods is to reduce the computational time in performing a detailed simulation of a physical system. A sample large system that can benefit from such a reduction, is a combustion chamber that is fed by a large number of injectors. Detailed flow physics within each injector is coupled with their interactions via the combustion chamber, as well as through the manifold. A fine mesh resolution is needed for accuracy, while the calculation of flow with turbulent chemistry introduces stiffness and the need for small time steps in marching procedures. If liquid propellants are considered, a further increase in complexity occurs in modeling liquid jet interfaces, atomization and so forth.

Reduced models for complex systems such as the above can be viewed as surrogate models of the system that can be used for repeated evaluations when parametric studies are desired. We identify four application areas in combustion dynamics where such models can be effective:

(i) Optimization, control and stochastic modeling of the combustor. A "light-weight" reduced model of a combustor can be used in performing a rapid exploration of a design space using a range of initial and boundary conditions with nonlinear interactions. A stochastic model can be then afforded for the system (see, e.g., ref [5]), which is otherwise too expensive to construct.

(ii) Input to linearized analysis tools. Acoustic representations of combustors has been vital in the understanding of their dynamic behavior. In this context, there have been efforts to construct unsteady models of heat release and other flow phenomena using detailed simulations and use them in a loworder model of system dynamics (refs. [15, 22] address two variants of this approach).

(iii) As generalized interfaces between computational sub-domains. Using the example of an injector interfacing with a combustor (see fig [1a]), we note that a reduced model of the injector flow dynamics can be an effective boundary conditions with virtually no assumptions on the physics of wave interactions at the boundary. If this can be performed in efficiently in an error-controlled manner, it can save computational burden in performing expensive multi-injector calculations (e.g., ref. [23]).

(iv) Representation of a multi-component system as a collection of surrogate models. Reduced Basis Models (RBMs) can interact with one another to solve problems of practical interest, as has been shown, for instance, in Huynh et al. [16]. This can provide opportunities to interface sub-domains of combustion chamber with one-another, with reduced models representing each sub-domain, and a boundary procedure that connects them using models such as in (iii) above. Figure [1b] shows a stream-tube-like domain decomposition, with reduced models associated with individual injectors in a large combustor with mutual coupling.

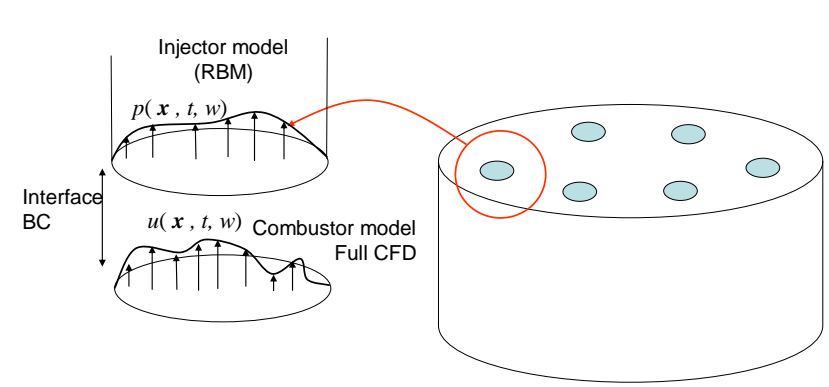

(a)

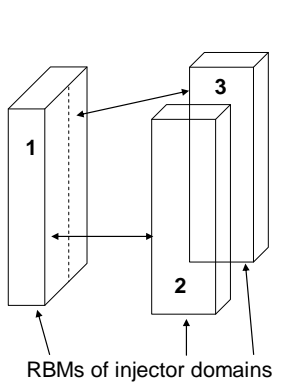

RBMs of injector domain

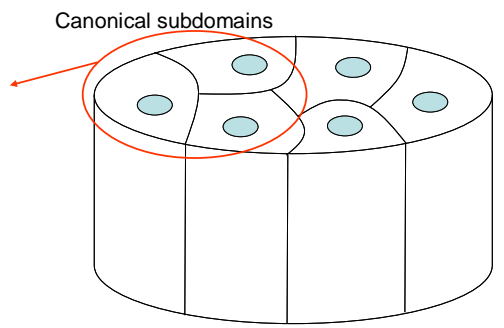

(b)

Figure 1: (a) Integration of injector sub-system model with combustion chamber, (b) Integrated operation of multiple reduced models as subdomains within a large combustor

Much of the mathematical applications in these areas have focused on systems governed by linear differential equations, or with simple polynomial (typically quadratic) nonlinearity. RBMs based on finite element formulations of steady, as well as time-dependent parameterized PDEs have been considered extensively in the literature ( refs. $[11,20]$ ). Recent advances (e.g., GNAT ${ }^{6}$, DEIM $^{8}$ ) show much promise for model reduction efforts in nonlinear dynamical systems. In this paper we present a sequence of developments in our efforts to apply these techniques to the equations of gas dynamics, leading in a sequence of steps, to the problems of turbulent combustion. 


\section{Mathematical Apparatus}

We begin by considering the time-dependant conservation equations of turbulent chemistry written in vector form:

$$
\frac{\partial Q}{\partial t}=F(Q, \vec{\mu})+W(Q, \vec{\mu})
$$

where $Q=\left[\begin{array}{lllll}\bar{\rho} & \bar{\rho} \tilde{u}_{i} & \bar{\rho} \tilde{E} & \bar{\rho} \tilde{Y}_{k}\end{array}\right]^{T}$ is the state vector of conserved mass momentum, energy and species density, $F$ is the flux vector and $W$ is a source term such as species production rate (see, e.g., ref. [12]). The state vector is a function of position, time and a vector of parameters, $\vec{\mu}$. These parameters could be global flow parameters such as flow Reynolds number, mass flow rate of fluid and oxidizer, swirl, forced pressure perturbation frequencies and so forth. When discretized, $Q$ and $F$ are replaced by their discrete equivalents based upon an appropriate numerical scheme. The number of unknown quantities in such a system is proportional to $\mathrm{K}$, where $\mathrm{K}$ is the number of grid points in the computational domain. A reduced basis model of this system is a low dimensional representation, with far fewer unknowns, say $\mathrm{N}$, such that $\mathrm{N} \ll \mathrm{K}$. It is to be noted that this representation will not linearize the system, or perform other such simplifying approximations of problem physics. Rather, a formal mathematical procedure is performed which minimizes the error incurred in approximating the solution in terms of carefully selected basis functions.

Reduced order approximations based on the proper orthogonal decomposition (POD) and its many variants have been widely used in CFD (see, e.g., Ito et al. [18], Iollo et al [17], Rowley et al [21], Bui-Thanh et al [5]), and to a limited extent in combustion dynamics (e.g., refs $[14,15]$ ). Attemts to date in combustion studies with reduced order modeling have focused on (a) developing modal analysis (POD, DMD, etc.) to post-process the results of simulations or experiments, or (b) using modal analysis to create control laws upon transferring flow information to a simplified systems of perturbation-derived equations. POD methods themselves have to be modified and implemented in special ways to handle multiple parameters in a given system.

Nonlinearity places limitations on the accuracy, stability, and efficiency of model reduction procedures. A pure POD-Galerkin based approach has been shown in some instances to result in wrong asymptotic steady state due to insufficient dynamic range in the basis. While simple polynomical nonlinearities have been effectively treated using simple extensions to linear methods, those approaches are inapplicable to general compressible flows as in the present context. During the last few years, methods based on empirical interpolation methods (EIM) [2], also known as discrete empirical interpolation methods (DEIM) [8], have emerged as efficient tools to overcome this challenge and recover models with an efficiency comparable to that of models for linear problems. A different approach, GNAT, which uses an iterative solution to the time-stepping of the reduced nonlinear discretized equation set has been shown in recent times to have a number of benefits in nonlinear dynamics. In the following, we focus on methods derived from the DEIM approach, presenting its basic features below. Details of our experiments with variants of this method have been presented elsewhere (ref [25]).

\section{An summary of DEIM-type model reduction methods:}

Let us restate the system of conservation laws above, this time expressing the right hand side as a sum of linear and nonlinear terms, as follows (we ignore the parameter dependence for the present) :

$$
\frac{\partial Q(x, t)}{\partial t}=-\frac{\text { Linear }}{A Q(x, t)}-\frac{\text { Non-linear }}{F(Q(x, t))}
$$

where $A Q$ is a sum of linear fluxes and source terms, and $F$ is the nonlinear flux of these quantities (including source terms which accounts for species production and depletion rates and so forth). $Q$ is then approximated as $Q_{R B M}$ using a modal expansion (Galerkin technique) in terms of modes $\psi_{n}$ as: $Q_{R B M}(x, t)=\sum_{n=1}^{N} Q_{R}(t) \psi_{n}(x)$, where the modes are obtained such that this expansion reduces an appropriately defined numerical error: $\left\|Q(x, t)-Q_{R B M}(x, t)\right\|$ as the number of modes in this expansion is increased. The coefficients $Q_{R}$ are obtained by solving a system of $1^{\text {st }}$ order ordinary differential equations (stated here for the case when the modes are orthogonal to one another):

$$
\left.\frac{d Q_{R}(t)}{d t}=-A Q_{R}(t)-\psi_{n}(x) F\left(\psi_{n}(x) Q_{R}(t)\right)\right)
$$


The calculation proceeds in two parts: The first is performed "offline", when the full set of equations is solved at a set of equal intervals in time, and solution snapshots are assembled. These snapshots can be used to construct a set of orthogonal basis functions $\psi_{n}$ using techniques such as proper orthogonal decomposition (POD). (See, e.g., refs. $[17,18]$ for details about this procedure). These basis functions provide a representation of the solution snapshots in the following sense. If we consider a matrix $V_{n}$ whose columns are the basis functions, we can "project" the set of snapshots $Q(x, t)$ into a space spanned by these basis functions, and write:

$$
Q(x, t) \approx V_{n}(x) Q_{R}(t)
$$

This expression is approximate if we do not include the entire set of basis functions. When we select the basis functions with the largest eigen values, we effectively compress the snapshot data into a lower dimensional representation. The discrete version of the projected ODE set will be (we assume there are $\mathrm{N}$ modes and $\mathrm{K}$ cells in the computational domain):

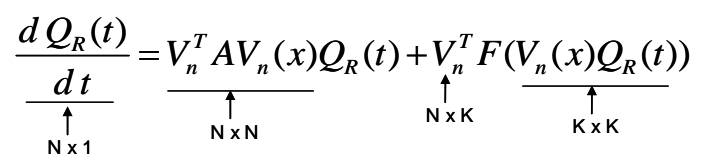

The second part in RBM is an "online" calculation which involves the solution to the ODEs mentioned above. These equations are solved to obtain temporal coefficients of the basis functions, which by requirement are far fewer than the full number of unknowns, as mentioned earlier. An efficiency problem arises immediately due to the nonlinear fluxes. The last term in the above equation shows that the operation count to evaluate the nonlinear flux is unchanged from the full model and scales with the number of cells in the domain K. Chaturantabut et al. [8] present a method to overcome this inefficiency, developing a Discrete Empirical Interpolation Method (DEIM), which has been used successfully in more general RBM scenarios by Drohmann et al [10]. Their approach is as follows.

Approximate the nonlinear flux in terms of modes $U(x)$ as follows: $F(Q(x, t)) \approx U(x) c(t)$.

In the above, the modes $U(x)=\left\{u_{1}(x), \ldots u_{m}(x)\right\}$ are obtained from a space spanned by the POD modes describing the nonlinear flux $F$. A greedy algorithm (Empirical Interpolation Method, EIM), selects a subset of this modal decomposition to reduce the size of the $\mathrm{F}$ vector to $\mathrm{m}(\ll<\mathrm{N})$. We simply state the final expression for the reduced model (where $P$ is a selection matrix which picks the elements of $U$ above that are needed):

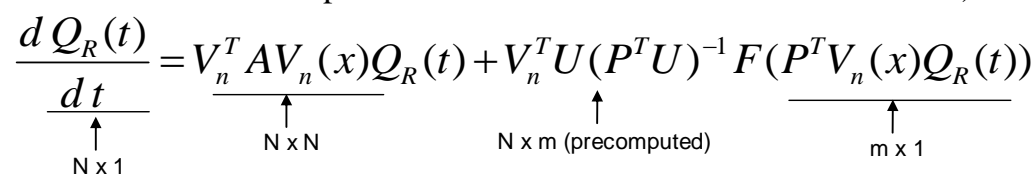

This last equation is the final form of the reduced basis model of the nonlinear conservation law. There are several possible variants of this approach, as we discuss in ref [25]. The method can be extended to problems with multiple parameters, wherein a POD-type approach is used to model (compress) the time variation, and a greedy algorithm is used to determine the modes that are parameter dependent. Time varying parametrized functions can be represented at discrete instances of time $t_{k}$, where $k=1 \ldots \mathrm{K}_{\max }$ as time samples are selected in the range $t_{k}=\{0 \ldots \mathrm{T}\}$ using a slightly rewritten version of the earlier expansion: $Q^{k}(x, \mu) \approx V_{n}(x) Q_{R}^{k}(\mu)$, where $k$ is a time-index, and the amplitudes $Q_{R}$ are now time trajectories for a given parameter vector $\mu$. Figure [2] below illustrates a low dimensional representation of a system using a series of trajectories with parameter and time variation.

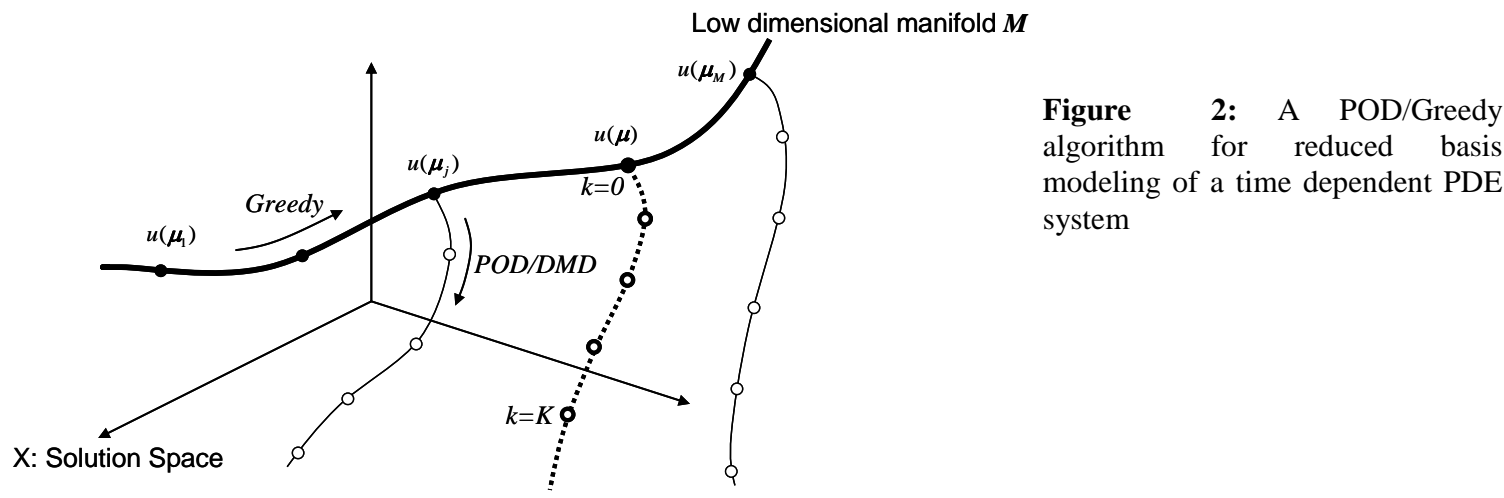

4

American Institute of Aeronautics and Astronautics 


\section{Case Studies}

In developing applications of the above ideas to problems in gas dynamics, we followed a path beginning from scalar conservations laws (scalar wave equation, Burgers' equation), progressing in complexity into solvers for 1-D Euler equations, 2-D and 3-D Navier-Stokes equations, leading up to the LES solver developed at Georgia Tech (LESLIE-3D) for turbulent combustion. We present here some highlights of this development.

\section{1-D Shock Tube Flow}

We basis functions using POD and EIM approaches and investigated variants of the DEIM approach which combine these functions to form the expansions of the state vector $Q$ and flux vector $F$ discussed in the previous section. Figure [3] shows a successively refined (increasing number of basis functions $M$ to compute the nonlinear flux). We selected Sod's shock tube problem for this study, where an initial discontinuity in density and internal energy drives the flow. We find, as expected, that successive addition of basis functions improve solution quality in general, but as the discontinuity becomes steeper (higher density ratio), anomalous behavior exists which creates instabilities. This phenomenon is shown in figure [4], where we see an under-resolved $(M=20)$ and an over-resolved $(M=250)$ solution to the same shock tube problem as in figure [3]. In the case of the under-resolved or under-fitted solution, there is inadequate spectral content in the solution and wave dynamics are completely inaccurate. In the situation where there are too many basis functions to describe the nonlinear flux, high frequency oscillations dominate and destroy the solution. To avoid an empirical determination of the number of basis functions, we expect that a proper choice will consist of an iterative scheme with oscillation bounds, which we are still developing.
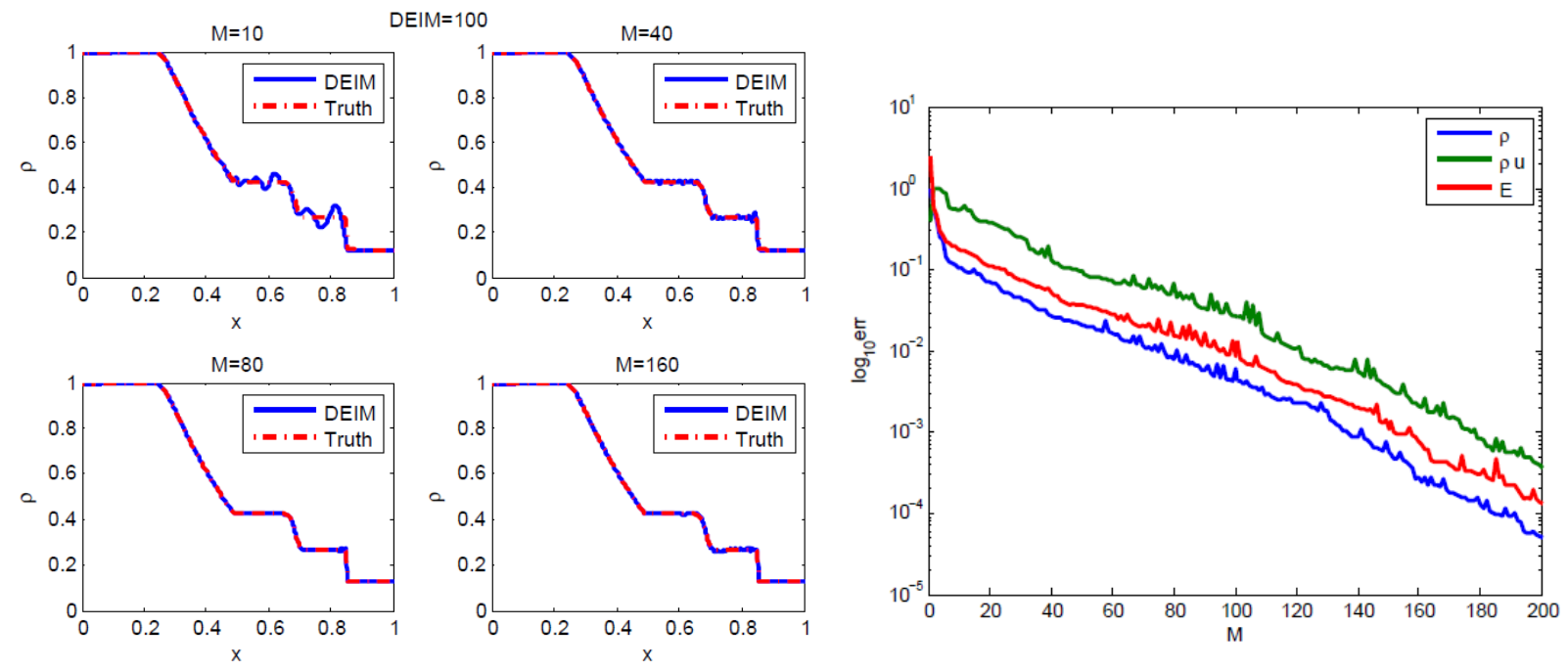

Figure 3: DEIM model of Sod's shock tube flow showing spectral convergence as the number of modes is increased
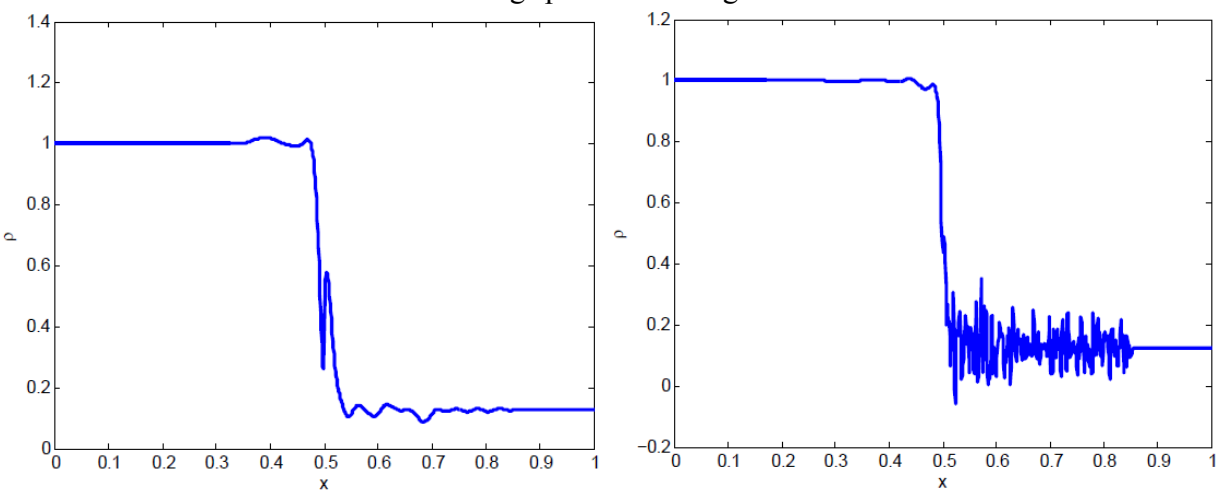

Figure 4: $M=20$ and $M=250$ profiles for computed values of density in the shock tube problem from fig [3]

Two variants of the DEIM approach were primarily studied. These were named:

(a) SEIM (Semi Discrete Empirical Interpolation Method,) where the basis functions for flux F are selected using the EIM method from snapshots of F rather than from the POD of those snapshots as in DEIM, and, 
(b) FEIM (Full EIM) where EIM is used to generate the basis functions for both the state vector Q as well as flux F.

We are still working with these methods in different scenarios to determine their relative merits. For the present, we present a comparison of convergence for the mass, momentum and energy fluxes of one variant of the 1D shock tube problem using these approaches, in figure [5]. The FEIM method appears superior among the three here.
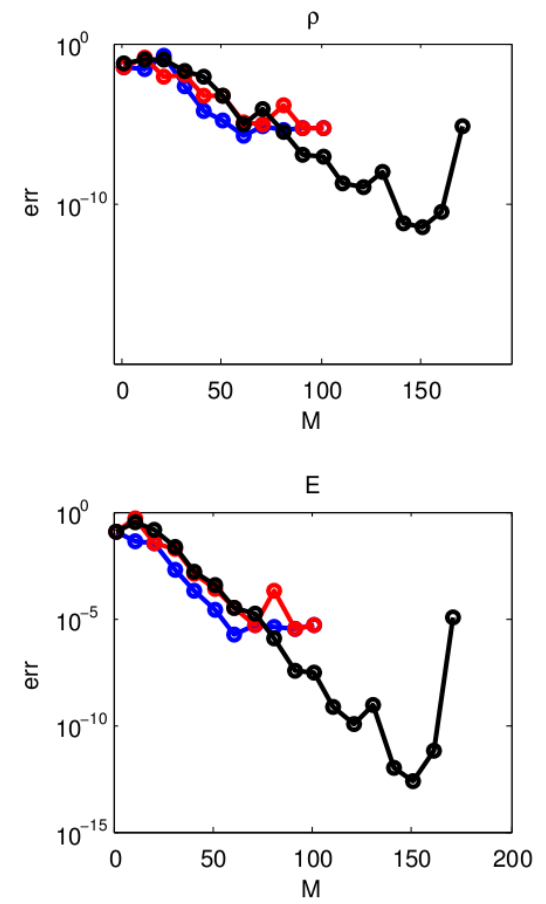

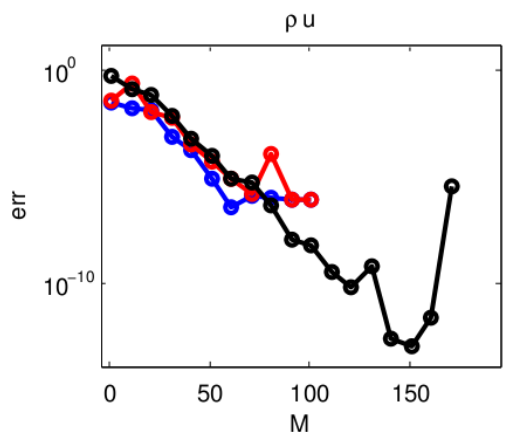

Figure 5: Comparison of error convergence in the DEIM, SEIM and FEIM methods.

\section{2-D Cylinder flow in a channel}

We applied the EIM and POD methods to study basis function generation for a 2D cylinder flow. Reduced models based on the above approaches were used to study this case. However, due to smoothness and simple periodicity inherent in these solutions, performance seemed rather similar. Modes and convergence are briefly illustrated below.

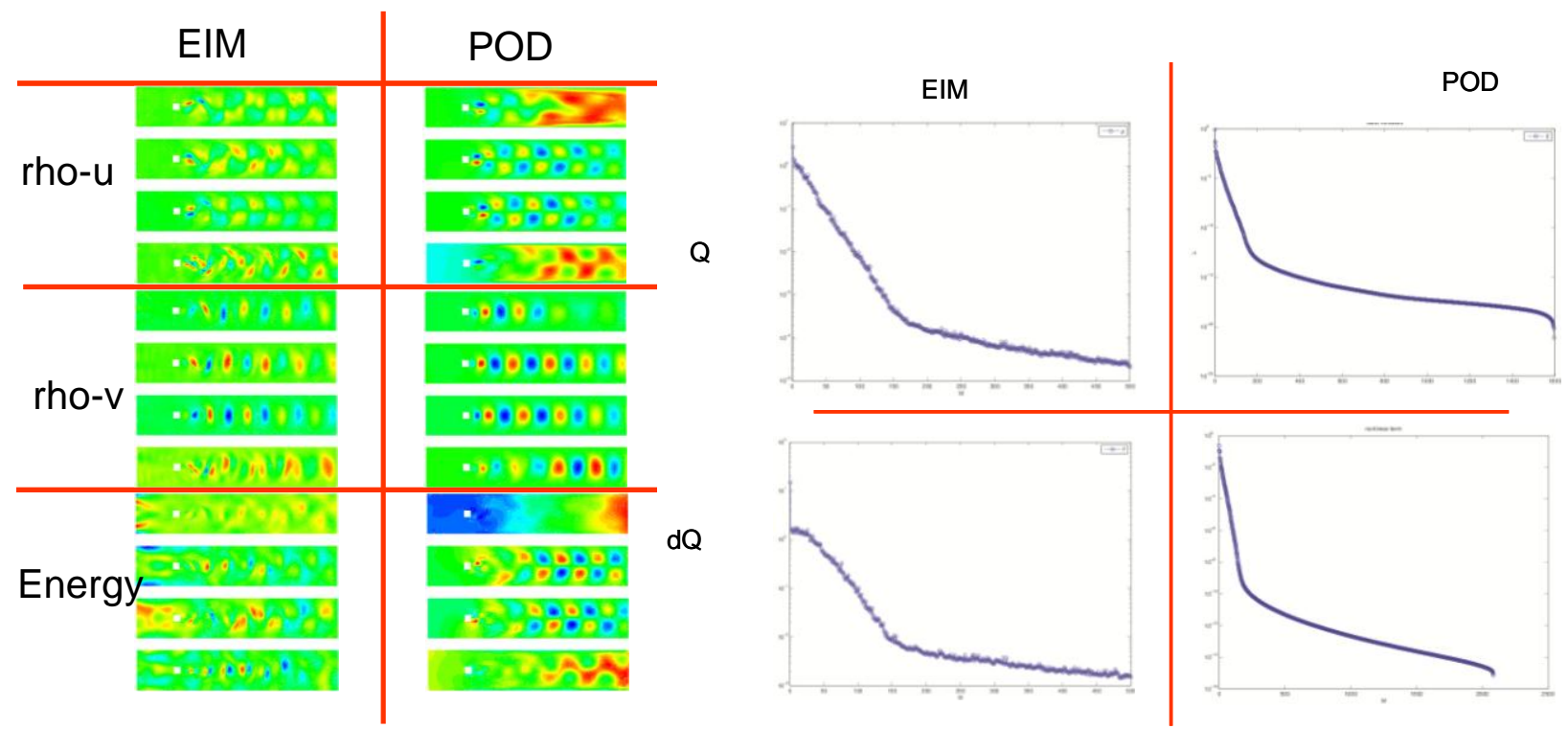

Figure 6: Basis functions (modes) generated from EIM and POD for cylinder flow, with corresponding error (EIM) and eigen value plots for the state vectpr Q and flux F (here called dQ) 


\section{Specification of initial and boundary conditions}

For a time advancing solution, the initial condition must be specified by projecting the desired value over the set of basis functions selected. This projection provides an initial set of amplitudes which are then marched in time in the RBM procedure. Figure [7] shows an LM6000 combustor calculation with swirling flow in the injector that has been projected into the first 10 modes with a nearly identical profile (axial velocity contours are shown here). Homogeneous boundary conditions are satisfied by the POD modes (linear combinations of the snapshots satisfy homogeneous BCs). Non-homogeneous BCs can be subtracted out while generating the basis function set and added during the RBM solution procedure. Alternatively, as in ref [13], an additional constraint can be added to the reduced model that enforces the conditions (sometimes as a penalty term).
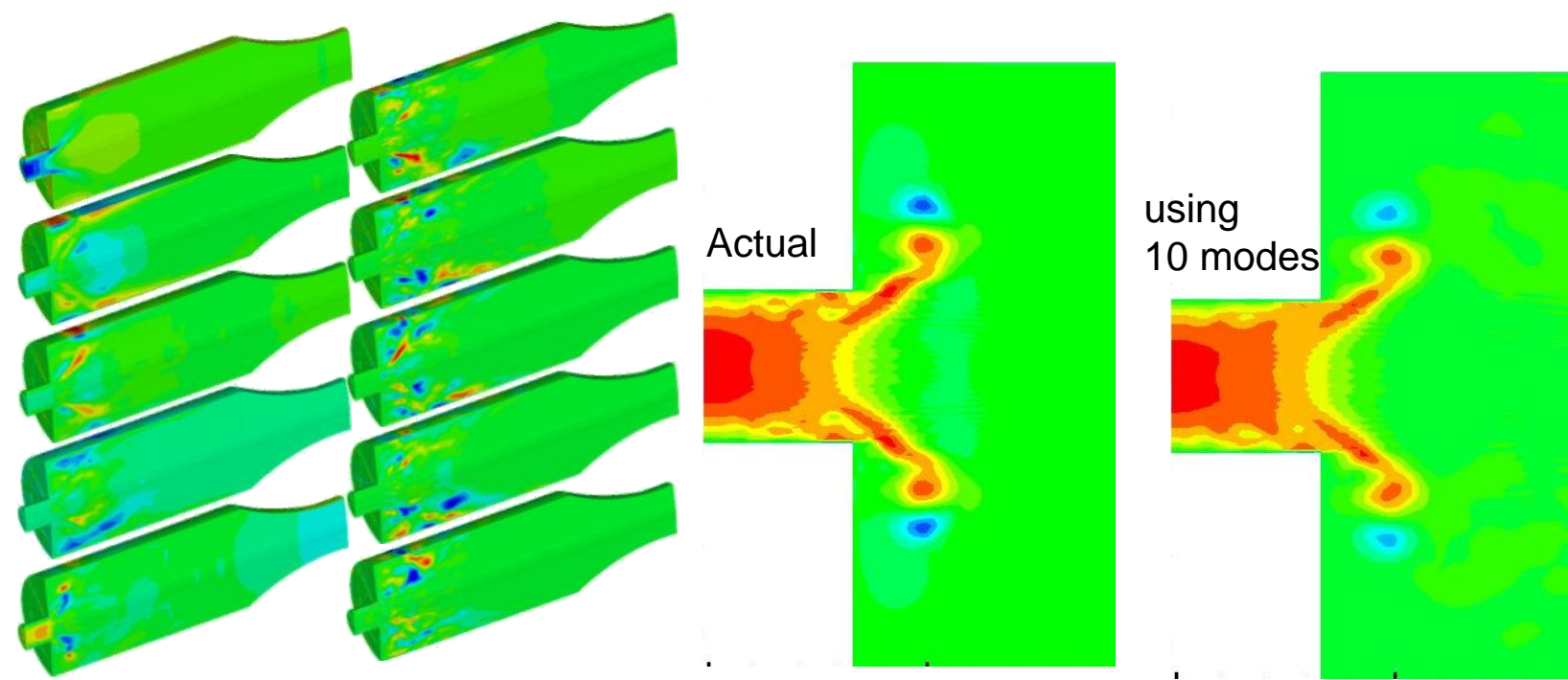

Figure 7: First 10 POD modes of axial velocity in an LM6000 combustor and a projected initial condition

\section{A 3D flow with hydrodynamic instability}

We use the case studied by Cantwell et al (ref. [7]) where a tangential hydrodynamic instability was observed in a suddenly (1:2) expanded low speed cylindrical flow. The geometry and flow configuration is shown in figure [8]. Full CFD solutions were computed and a reduced model of the evolution of the flow was developed. Flow velocity at the inlet was $29 \mathrm{~m} / \mathrm{s}$ (Mach 0.1). When the flow settles to a quiescent state, a random perturbation in the tangential component of the velocity is given as suggested in ref [7]. Flow Reynolds number w.r.t. diameter of the feeding channel was set at 600 .

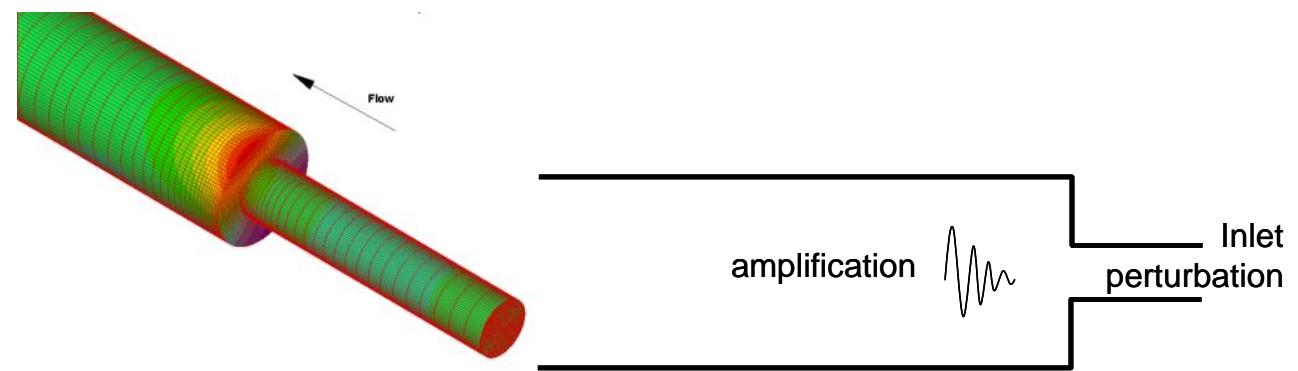

Figure 8: 3D channel geometry from ref [7] using a 763,248 element mesh

The input perturbation amplifies and results in oscillatory limit cycle behavior of the flow. Figure [9] shows a comparison of the reduced model vs. full model contours of tangential velocity. Predicted amplitudes from the RBM procedure are shown for the first three modes of density. This simulation was performed using HyPerComp's internal CFD solver. 

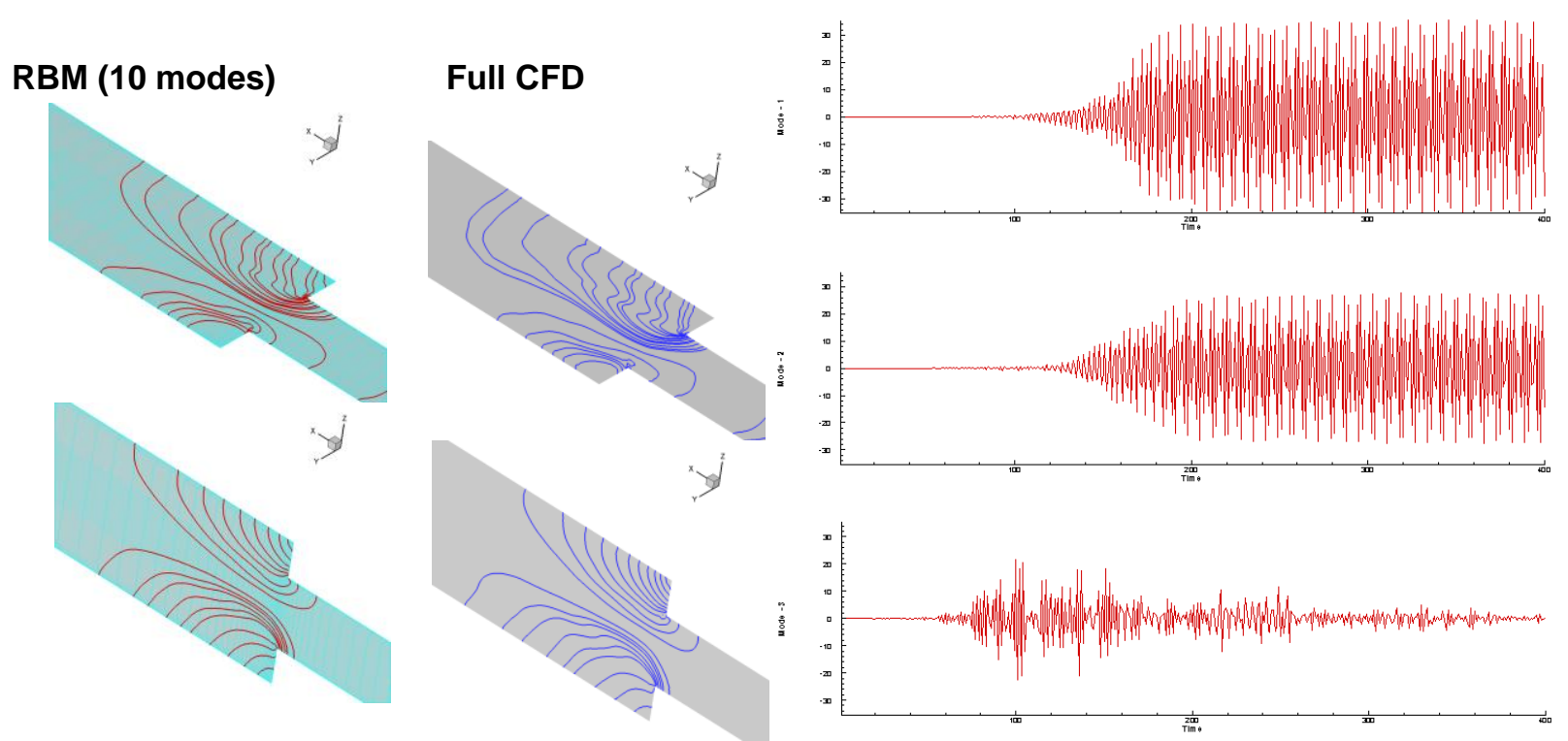

Figure 9: RBM vs. full CFD flow solution and amplitudes of the first three density modes in time

\section{LOX-post injector flow dynamics}

We present here results from a model reduction study of a LOX-post injector, earlier discussed in ref [1]. 13002 hexahedral cells were used to grid a $5^{\circ}$ sector of the cylindrical geometry with symmetry BCs on the lateral surfaces, as shown in figure [10]. The Peng-Robinson equation of state was used to model the supercritical flow of liquid Oxygen. A low speed inflow $(0.4 \mathrm{~m} / \mathrm{s})$ was converged to steady state, at which time forced pressure oscillations were imposed at the outflow boundary. The imposed oscillations were $2.5 \%$ of the mean pressure. Longitudinal mode instability occurs in this flow due to these oscillations. Due to the physical complexity in this model, it is computationally intensive. A sample run took 47 hours on 32 cores to converge to a limit cycle. Reduced models of this problem only take a few seconds to complete for a given parameter value.

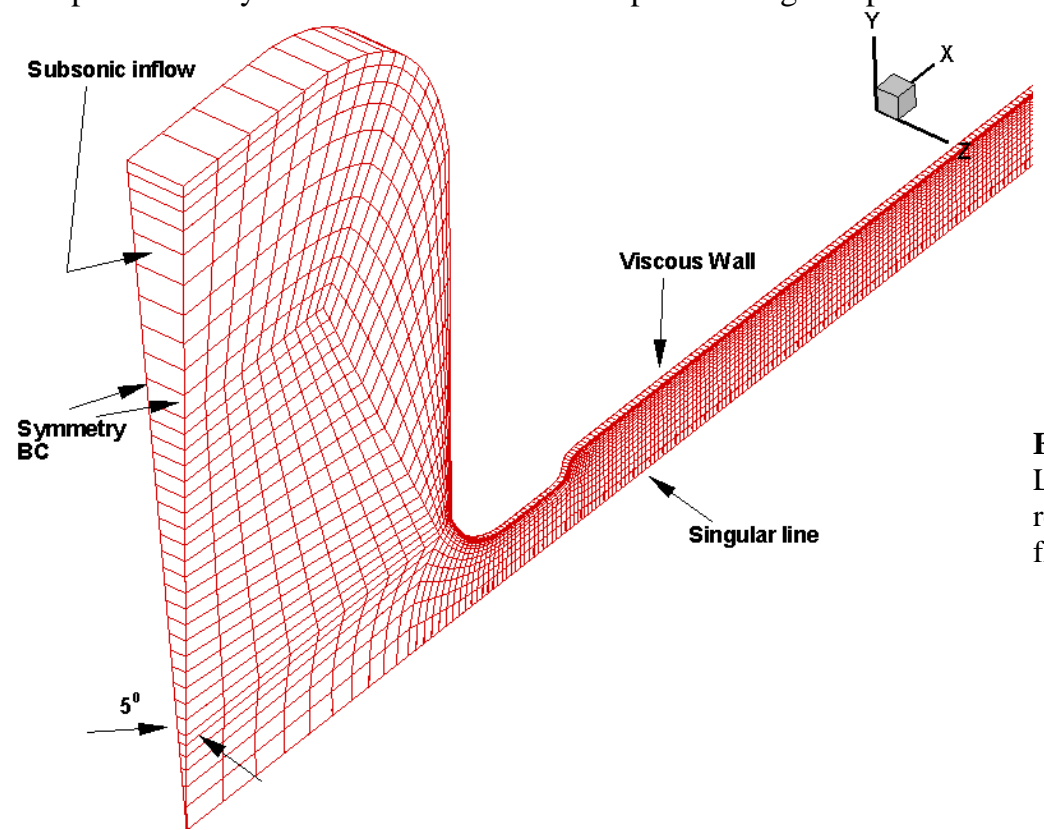

Figure 10: A mesh showing the LOX post injector and an upstream reservoir region considered in the flow analysis

Figure [11] shows the injector response function, defined as the ratio of computed mass flow rate to applied pressure fluctuations in the frequency domain. In order to build an RBM of this model using forcing frequency as a parameter, we define a "learning set" comprising of the frequencies listed in figure [11], based on full CFD analysis. In general, this is not possible, and an error estimator, or a "light-weight" solver is needed to span the parameter space for points where error control is desired when a certain set of basis functions are used. Here, we define a parameter-dependent basis from computed solutions at these points. 


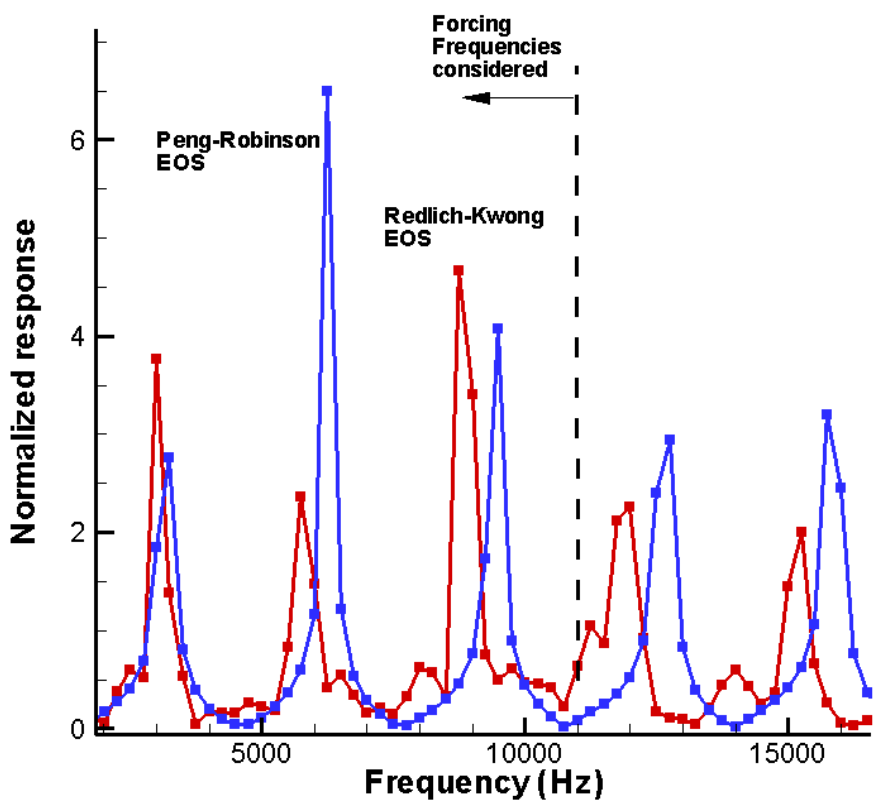

Frequencies sampled $(\mathrm{Hz})$ :

2250

2750

3250

3750

4750

5750

6250

6750

7250

8000

9000

9500

10000

10500

Figure 11: System response derived from detailed CFD, showing sample points considered for EIM

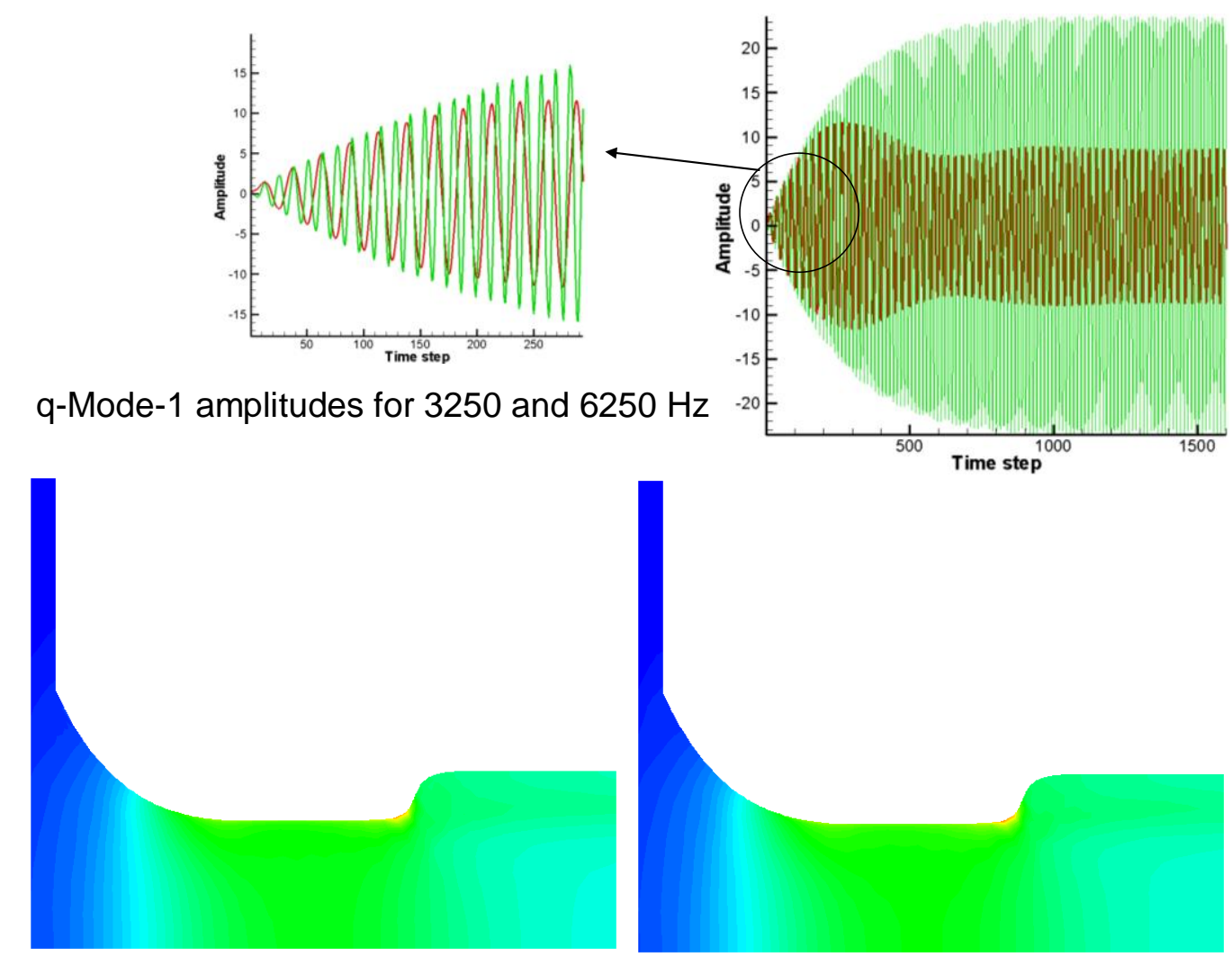

Figure 12: Mode-1 amplitudes of density predicted from RBM solution for two resonant frequencies (above) and solution snapshots comparing full solution (left) and RBM solution (right)

Figures [12] show computed amplitudes of the first density mode from the reduced model of the LOX injector geometry. Snapshots are seen to represent the solution accurately, while amplitude variations are indistinguishable from the true amplitude variations from a full CFD simulation of this problem. 


\section{An interface model at a sudden expansion}

As stated earlier we are interested in a reduced basis representation for the flux that interfaces two domains (figure [13]), as in the case of an injector feeding a combustor. The goal is to replace a detailed model of the flow phenomena in the injector by its surrogate in a full CFD calculation. This problem exhibits steady as well as timedependent bifurcation phenomena which depend on Reynolds number as a parameter (e.g., ref [3]) as well as others, depending on the actual case. Once such a representation is derived, we essentially have a boundary condition which preserves in a nonlinear sense the dynamical information, as will be evidenced by the model's ability to reproduce stability data as presented in ref. [3]. This problem is important, since it can lead to a practical solution to the multiinjector system including crucial details of injector dynamics (see refs. [3,4,19] for the importance of including injector dynamics in this calculation; note that due to affordability for the most part, as in ref [23], the computational domain is truncated at this boundary).
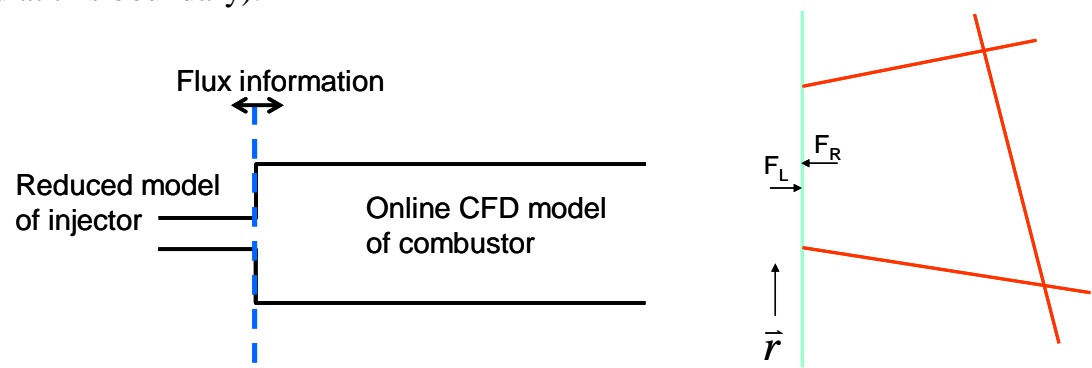

Figure 13: Model problem for interaction between sub-domains and a finite volume cell showing left and right interfaces

We consider the following finite volume expression in a cell that has a boundary interface being modeled by RBM, sketched in figure [13]:

$$
y_{i}^{n+1}=y_{i}^{n}+\frac{\Delta t}{\Omega}\left(\sum_{j \neq j R B M} F_{j}+\sum_{j=j R B M} F_{j}\right)
$$

Flux at the left side of the interface is to be approximated:

$$
F_{L}=F_{L}\left(t, \vec{r}, F_{R}, \vec{\mu}\right)
$$

Presently, we seek the following approximation in terms of time and a parameter vector (which could comprise of mass flow rate, viscosity and so forth):

$$
F(x, t ; \mu)=\sum_{j=1}^{N_{p}} \sum_{i=1}^{N_{r}} \sum_{k=1}^{N_{k}} \alpha_{i k}(t) \phi_{i}\left(x ; \mu_{j}\right) \beta_{j}(\mu)
$$

In this interpolation law, $N_{p}-1$ is the degree of the Lagrange polynomial used to cover the parameter space (in this case, the Reynolds number). $\mu_{j}$ are Gauss-Lobatto points and $\beta_{j}$ is the Lagrange polynomial. $N_{r}$ is the number of POD basis functions $\varphi_{i}\left(x ; \mu_{j}\right)$ for each $\mu_{j} . N_{k}-1$ is the degree of polynomial to match the history of the coefficients of the POD basis. In our tests we found the values of $N_{p}=3, N_{r}=14, N_{k}=14$ to adequately represent the time evolutions of the solutions at this interface for an order of magnitude variation in Reynolds number, starting from a no-flow initial condition. The sample domain we select for this problem is in $2 \mathrm{D}$ in order to rapidly converge on the mode selection process. The following figures show the computational domain and error behavior.

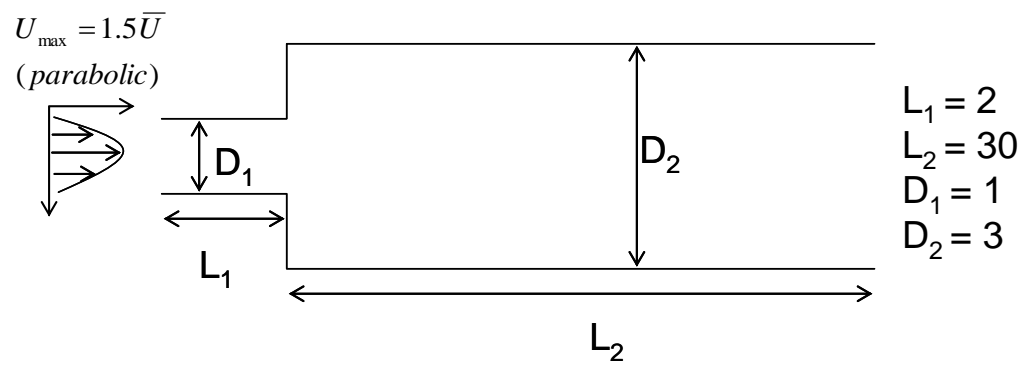

Figure 14: 2D channel with sudden expansion - a case study 

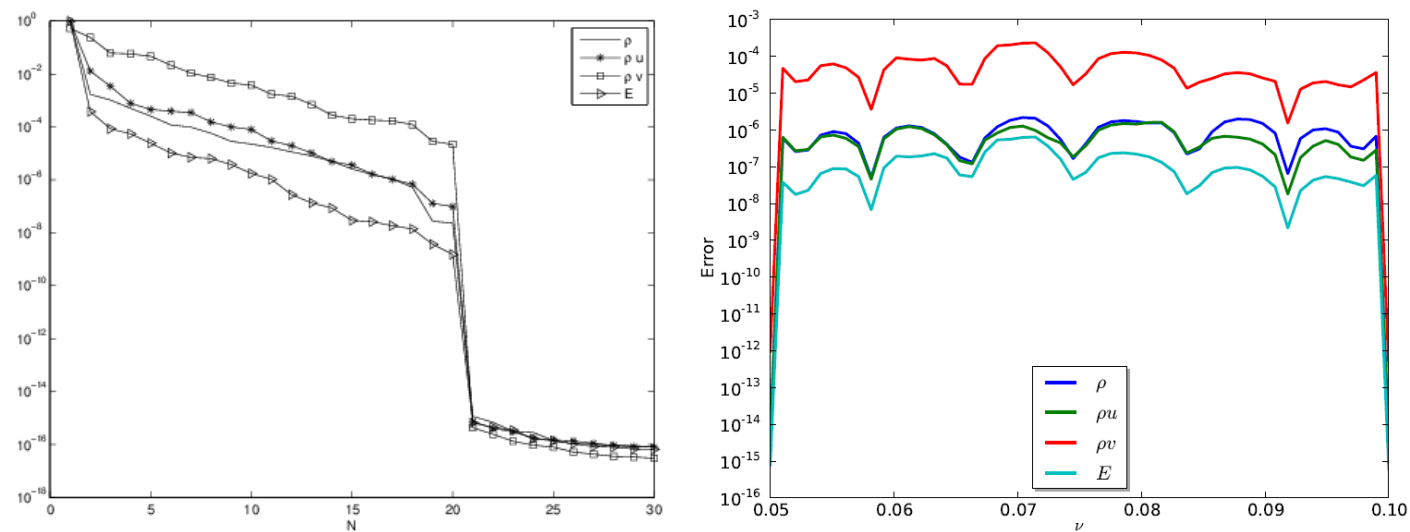

Figure 15: POD eigen values for time variation (left) and max interpolation error in parameter space (right)

\section{Prospects for model reduction in realistic combustor studies}

We begin by studying the eigen-value content in POD modes of turbulent combustion in realistic combustor calculations. A case of much interest is the continuously variable resonance combustor (CVRC) experiment from Purdue University (ref. [24]), due to the detailed measured data that is available and the many simulations performed to understand it. An LES study was performed using the Georgia Tech LESLIE-3D code for this problem (e.g., ref [12]). Figure [16] shows a modal analysis of an axi-symmetric calculation of the CVRC combustor. The eigen values of heat release show an encouraging decline until about the first 6 modes after which the error drops relatively slower. We determined that about 100 modes are needed to reconstruct the entire solution based on simple reconstructions of the data.

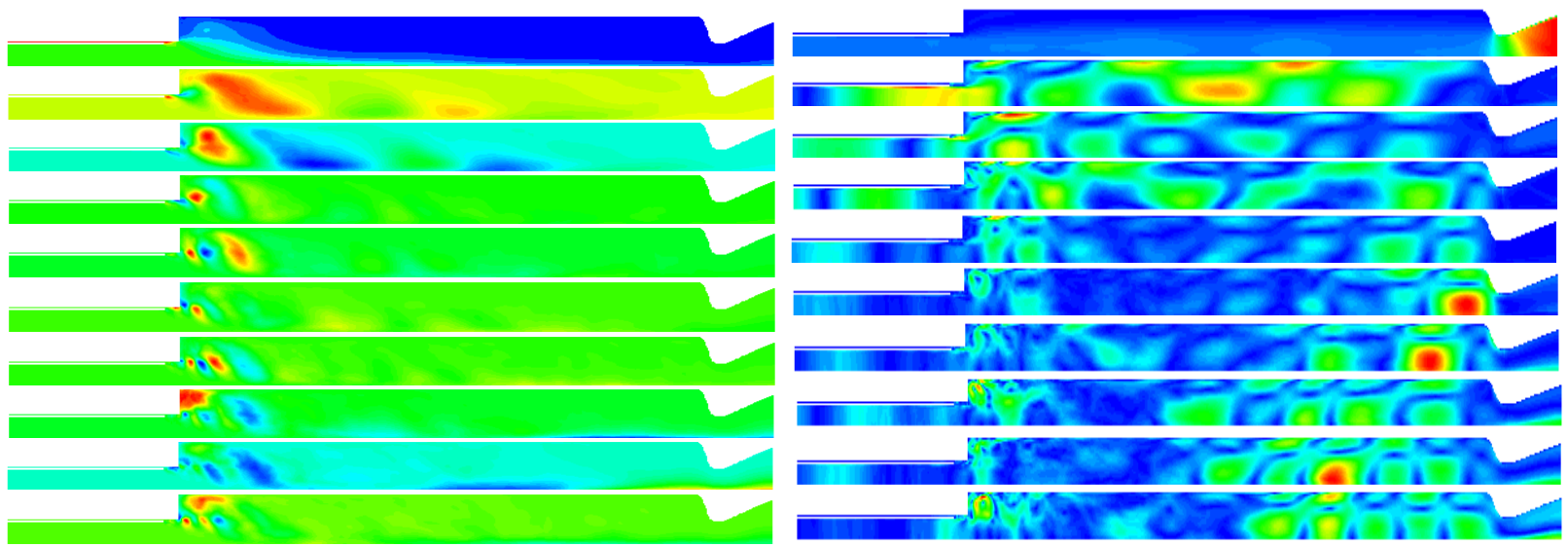

Figure 16: Heat release parameter (left) and velocity magnitude (eight) modes computed for the CVRC combustor

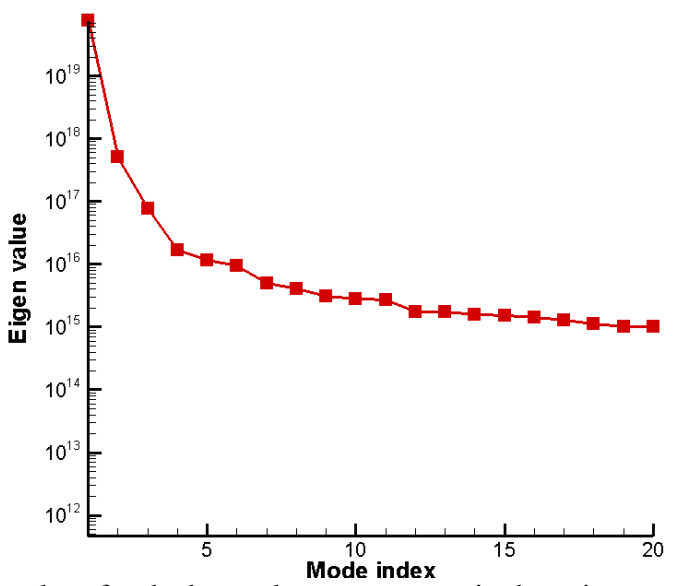

Figure 17: POD Eigen values for the heat release parameter in the axisymmetric CVRC calculation 


\section{Reduced models of the CVRC injector interface}

A 3D version of the CVRC combustor has recently been performed using LESLIE-3D. Sample density contours are shown in figure [18], highlighting the interface between the injector and the combustor. We note that a significant amount of flow activity occurs ahead of the injector, thereby placing special demands on the accuracy of the reduced model. There are two components to this sort of model reduction (as suggested by the earlier discussion on the interface sub-models). The first is using POD to approximate time trajectories and the second is to include parametric variations. In this case, the parameters we will be interested in primarily include the length of the injector and mass flow rates. Figure [18] shows the rapid decline in POD eigen values for the injector interface, thus establishing the low dimensionality of the data. Figure [19] shows approximations of interface pressure based on 8 and 20 modes. Parametric studies are presently underway.
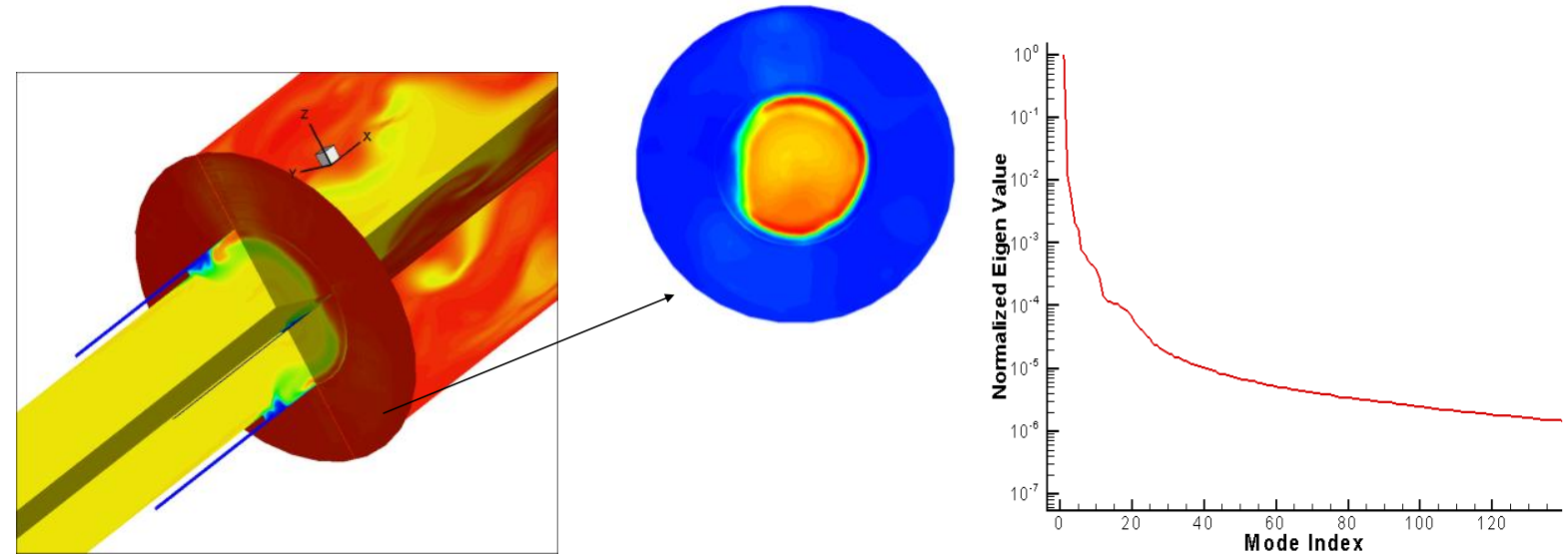

Figure 18: The CVRC injector showing an injector interface boundary for model reduction and POD eigen values
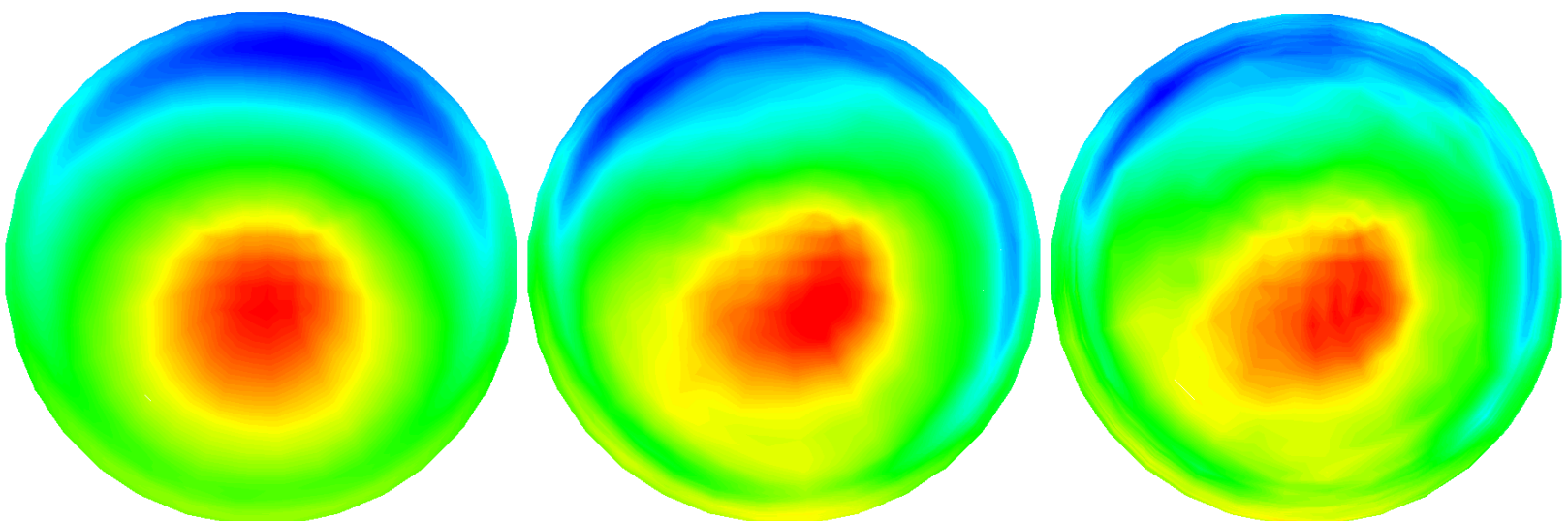

Figure 19: Low dimensional (8 modes, 20 modes) representations of the true pressure distribution (right) 


\section{Summary}

We are pursuing the application of recent developments in reduced basis methods to challenging problems in combustion dynamics. Model reduction of this nature has the potential to reduce computational effort and place complex simulations needed in the design of future engines within reach. This work is an initial attempt at exploring the scope of these methods and demonstrating initial feasibility. We anticipate that future publications will follow shortly with further developments in the methods as well as applications.

\section{Acknowledgments}

The work is funded in part by NASA Glenn Research Center under a NASA NRA (Dr. N-S. Liu, PM), grant NNX11AI22A, Air Force Office of Scientific Research (Dr. Mitat Birkan, PM) under grant FA9550-12-1-0156 and a Phase-I AF-STTR project (Dr. Douglas Talley, PM) under contract FA9300-13-M-1501. Thanks to Srikant Srinivasan and Jason Hamilton (Georgia Tech) for help with CVRC simulations.

\section{References}

1. Aithal, S.M., Liu, Z., Jensen, R.J., Hinerman, T.D., Lynch, E.D., "Nonlinear injection transfer function simulations for liquid propellants," AIAA paper 2008-4742, 2008

2. Barrault, M., Maday, Y., Nguyen, N.C., Patera, A.T., "An empirical interpolation method: application to efficient reduced-basis discretization of partial differential equations," C.R. Math. Acad. Sci. Paris Series I, V. 339, pp. 667-672, 2004

3. Battaglia, F., Tavener, S.J., Kulkarni, A.K., Merkle, C.M., "Bifurcation of low Reynolds number flows in symmetric channels," AIAA J., V. 35, No. 1, pp. 99-105, January 1997

4. Bazarov, V.G., Yang, V., "Liquid-propellant rocket engine injector dynamics," J. Prop. Power, V. 14, No. 5, pp. 797-806, 1998

5. Bui-Thanh, T., Willcox, K., Ghattas, O., "Parametric reduced-order models for probabilistic analysis of unsteady aerodynamic applications," AIAA J., V. 46, No. 10, pp. 2520 - 2529, 2008

6. Carlberg, K., Farhat, C., Cortial, J., Amsallem, D., "The GNAT method for nonlinear model reduction: Effective implementation and application to computational fluid dynamics and turbulent flows," J. Comp. Phys., V. 242, pp. 623-647, 2013

7. Cantwell, C.D., Barkley, D., Blackburn, H.M., "Transient growth analysis of flow through a sudden expansion in a circular pipe," Physics of Fluids, V. 22, pg. 034101, 2010

8. Chaturantabut, S., and Sorenson, D., "Nonlinear model reduction via discrete empirical interpolation," SIAM J. Sci. Comput., V. 32, No. 5, pp. 2737-2764, 2010

9. Culick, F., "Unsteady motions in combustion chambers for propulsion systems," Technical report, RTANATO AGARDograph, AG-AVT-039, 2006

10. Drohmann, M., Haasdonk, B., Ohlberger, M., "Reduced basis approximation for nonlinear parameterized evolution equations based on empirical operator interpolation," SIAM J. Sci. Comput., V. 34, No. 2, pp. A937969,2012

11. Grepl, M.A., "Certified reduced basis methods for nonaffine linear time-varying and nonlinear parabolic partial differential equations," Math. Models and Methods in Applied Sciences, Vol. 22, No. 3, 2012

12. Guezennec, N., Masquelet, M., Menon, S., "Large Eddy Simulation of Flame-Turbulence Interactions in a LOX-CH4 Shear Coaxial Injector," AIAA paper 2012-1267, January 2012

13. Gunzburger, M., Peterson, J.S., Shahid, J.N., "Reduced-order modeling of time-dependent PDEs with multiple parameters in the boundary data," Comput. Methods Appl. Mech. Engrg.,V. 196, pp. 1030-1047, 2007

14. Huang, Y., Wang, S., Yang, V., "Systematic analysis of lean-premixed swirl-stabilized combustion," AIAA J., V. 44, No. 4, pp. 724-740, April 2006

15. Huang, X., Baumann, W.T., "Reduced-order modeling of dynamic heat release for thermoacoustic instability prediction," Comb. Sci. Tech., V. 179, No. 3, pp. 617-636, March 2007 
16. Huynh, D., Knezevic, D., Patera, A., "A static condensation reduced basis element method: approximation and a posteriori error estimation," Math. Mod. and Num. Analysis, 2011

17. Iollo,A., Lanteri,S., Desideri, J., "Stability properties of POD Galerkin approximations for the compressible Navier-Stokes equations," Theo. Comp. Fluid Dyn., V. 13, pp. 377-396, 2000

18. Ito, K., and Ravindran, S. S., "A reduced-order method for simulation and control of fluid flows,” J. Comp. Phys., V. 143, pp. 403-425, 1998

19. Jensen, R., Dodson, H., Claflin, S., "LOX/hydrocarbon combustion instability investigation," NASA contractor report: NASA CR-182249, 1989

20. Quarteroni, A., Rozza, G., Manzoni, A., "Certified reduced basis approximation for aprameterized partial differential equations and applications," J. Math. in Industry, Vo. 1, No. 1, pp. 1-49, 2011

21. Rowley, C.W., Colonius, T., Murray, R.M., "Model reduction for compressible flows using POD and Galerkin projection," Physics D, V. 189, pp. 115 - 129, 2004

22. Yao, Z., Gao, Y., Zhu, M., Dowling, A.P., Bray, K.N.C., "Combustion rumble prediction with integrated computational fluid dynamics/low order model methods," J. Prop. Power, V. 28, No. 5, pp. 1015-1025, 2012

23. Yoo, K.-H., Kim, J.-C., Sung, H.-G., Zhang, L., Yang, V., "Flow dynamics in combustors with multielement swirl injectors," AIAA paper 2011-786, 2011

24. Yu, Y.C., Sisco, J.C., Rosen, S., Madhav, A., Anderson, W.E., "Spontaneous longitudinal combustion instability in a Continuously Variable Resonance Combustor,” J.Prop. Power, V. 28, No. 5, pp. 876-887, 2012

25. Zhu, X., Hesthaven, J., Munipalli R., “Towards an efficient approach for model reduction via EIM," SIAM Conference on Comp. Sci. Engrg., 2013 